\title{
Interactions between model cell membranes and the neuroactive drug propofol
}

DOI:

10.1016/j.jcis.2018.03.052

\section{Document Version}

Accepted author manuscript

Link to publication record in Manchester Research Explorer

\section{Citation for published version (APA):}

Niga, P., Hansson-Mille, P. M., Swerin, A., Claesson, P. M., Schoelkopf, J., Gane, P. A. C., Bergendal, E., Tummino, A., Campbell, R. A., \& Magnus Johnson, C. (2018). Interactions between model cell membranes and the neuroactive drug propofol. Journal of Colloid and Interface Science, 526, 230-243.

https://doi.org/10.1016/j.jcis.2018.03.052

Published in:

Journal of Colloid and Interface Science

\section{Citing this paper}

Please note that where the full-text provided on Manchester Research Explorer is the Author Accepted Manuscript or Proof version this may differ from the final Published version. If citing, it is advised that you check and use the publisher's definitive version.

\section{General rights}

Copyright and moral rights for the publications made accessible in the Research Explorer are retained by the authors and/or other copyright owners and it is a condition of accessing publications that users recognise and abide by the legal requirements associated with these rights.

\section{Takedown policy}

If you believe that this document breaches copyright please refer to the University of Manchester's Takedown Procedures [http://man.ac.uk/04Y6Bo] or contact uml.scholarlycommunications@manchester.ac.uk providing relevant details, so we can investigate your claim.

\section{OPEN ACCESS}




\title{
Interactions between Model Cell Membranes and the
}

\section{Neuroactive Drug Propofol}

\author{
Petru Niga ${ }^{1 *}$, Petra M. Hansson-Mille ${ }^{1}$, Agne Swerin ${ }^{1,2}$, Per M. Claesson ${ }^{1,2}$, Joachim \\ Schoelkopf $f^{3}$, Patrick A. C. Gane $e^{3,4}$, Erik Bergendal ${ }^{2}$, Andrea Tummino ${ }^{5,6}$, Richard A. Campbell ${ }^{5}$ \\ and C. Magnus Johnson ${ }^{2 *}$ \\ ${ }^{1}$ RISE - Research Institute of Sweden, Chemistry Materials and Surfaces, Box 5607, SE-114 28 \\ Stockholm, Sweden. \\ ${ }^{2}$ KTH Royal Institute of Technology, Department of Chemistry, Division of Surface and \\ Corrosion Science, SE - 10044 Stockholm, Sweden. \\ ${ }^{3}$ Omya International AG, Baslerstrasse 42, CH-4665 Oftringen, Switzerland. \\ ${ }^{4}$ Aalto University, School of Chemical Engineering, Department of Bioproducts and Biosystems, \\ P.O. Box 16300, FI-00076 Aalto, Helsinki, Finland \\ ${ }^{5}$ Institut Laue-Langevin, 71 avenue des Martyrs, 38042 Grenoble, France \\ ${ }^{6}$ Eötvös Loránd University, Budapest 112, P. O. Box 32, H-1518 Hungary
}

Corresponding Authors:

*Petru Niga petru.niga@ @i.se and *Magnus Johnsson magnusj@kth.se 
ABSTRACT. Vibrational sum frequency spectroscopy (VSFS) complemented by surface pressure isotherm and neutron reflectometry (NR) experiments were employed to investigate the interactions between propofol, a small amphiphilic molecule that currently is the most common general anaesthetic drug, and phospholipid monolayers. A series of biologically relevant saturated phospholipids of varying chain length from $C_{18}$ to $C_{14}$ were spread on either pure water or propofol (2,6-bis(1-methylethyl)phenol) solution in a Langmuir trough, and the change in the molecular structure of the film, induced by the interaction with propofol, was studied with respect to the surface pressure. The results from the surface pressure isotherm experiments revealed that propofol, as long as it remains at the interface, enhances the fluidity of the phospholipid monolayer. The VSF spectra demonstrate that for each phospholipid the amount of propofol in the monolayer region decreases with increasing surface pressure. Such squeeze out is in contrast to the enhanced interactions that can be exhibited by more complex amphiphilic molecules such as peptides. At surface pressures of $22-25 \mathrm{mN} \mathrm{m}^{-1}$, which are relevant for biological cell membranes, most of thepropofol has been expelled from the monolayer, especially in the case of the $\mathrm{C}_{16}$ and $\mathrm{C}_{18}$ phospholipids that adopt a liquid condensed phase packing of its alkyl tails. At lower surface pressures of $5 \mathrm{mN} \mathrm{m}^{-1}$, the effect of propofol on the structure of the alkyl tails is enhanced when the phospholipids are present in a liquid expanded phase. Specifically, for the $\mathrm{C}_{16}$ phospholipid, NR data reveal that propofol is located exclusively in the head group region, which is rationalized in the context of previous studies. The results imply a non-homogeneous distribution of propofol in the plane of real cell membranes, which is an inference that requires urgent testing and may help to explain why such low concentration of the drug are required to induce general anaesthesia. 


\section{Introduction}

Both phospholipids and synthetic surfactants are amphiphilic molecules with distinct polar and non-polar regions, which cause them to self-assemble in aqueous solution. Many different selfassembled structures can form depending on the molecular shape and the interactions, as commonly described using the critical packing parameter $^{1}$. Double chained phospholipids typically have a packing parameter close to one, meaning that they preferentially self-assemble into planar structures, such as lamellar phases and biological membranes. In contrast, single chain surfactants in excess water tend to self-assemble into structures such as spherical or rodlike micelles. When such surfactants encounter a phospholipid membrane, they can be incorporated and as a result change the curvature of the membrane ${ }^{2}$. In the presence of sufficient surfactant(s), this often leads to the complete disruption of the biological membrane. Much is known about this important topic, as described in the excellent review by Heerklot $z^{3}$.

Many low molecular weight drugs, like classical surfactants, contain both hydrophobic and hydrophilic groups. However, due to their lower molecular weight and less distinct separation into hydrophobic and hydrophilic regions, the self-assembly of such drugs in aqueous solutions is less easy to describe in general terms. It is commonly observed that this type of drug interacts with phospholipid membranes. This is most often not the cause of the wanted effect of the drug, but it has rather been suggested to cause some of the unwanted toxic effects ${ }^{4}$. To gain more understanding of the interaction between such amphiphilic drugs with phospholipid membranes, we focus the present study on one particular drug molecule, propofol, which is predominantly hydrophobic but contains a hydrophilic $\mathrm{OH}$-group located in the middle of the molecule (see Figure 1). Propofol (2,6-bis(1-methylethyl)phenol) is a useful intravenous agent with several important applications ${ }^{5}$. It is probably most well-known for inducing general anaesthesia. Even 
though it is more than 150 years since general anaesthesia was introduced in medical surgery practice $^{6}$, the molecular mechanism by which drugs can produce this state is still under debate ${ }^{7-9}$, and occasions remain when standard dosing of such agents fails to induce a reliable amnesic state $^{10,11}$.

In the case of propofol, several mechanisms of action ${ }^{12,13}$ have been proposed, where potentiating $\mathrm{GABA}_{\mathrm{A}}$ (gamma aminobuthyric acid type A) receptors ${ }^{7}, 14,15$ is the most acknowledged one. The binding site of propofol to the $\mathrm{GABA}_{\mathrm{A}}$ protein is in the transmembrane region ${ }^{16}$, suggesting that the amphiphilic nature of propofol is of importance for its action, and the presence of propofol can possibly lead to perturbations of the molecular order of the lipid membrane ${ }^{17}$. For instance, Tsuchiya ${ }^{18}$ studied a series of structural analogues to propofol and found that propofol is the most effective in fluidizing model membranes.

Propofol has also been shown to have antioxidant, anxiolytic, analgesic, immunomodulatory and anticancer effects ${ }^{5,19}$. Thus, being widely used as an intravenous agent, it is of importance to have a thorough knowledge about how propofol interacts with cell membranes, and it has been suggested that it penetrates the lipid bilayer forming the plasma membrane ${ }^{20}$. However, it remains unclear how these interactions occur on a molecular level.

The location of a drug in a monolayer represents another important clue in its mode of interaction. A previous study on pre-mixed sample of propofol with a saturated phosphatidylcholine found that the drug lowers the compressibility of the monolayer, especially at low surface pressures, that it is located close to the head group of the phospholipid due to hydrogen bonding with the phosphate, and that at the same time it increases the order in the alkyl tail region ${ }^{17}$. 
The aim of our study is to obtain a more extensive molecular picture of interactions between propofol and different phospholipid membranes with respect to their fluidity. To this end, we have chosen to use model phospholipid monolayers at the air-water interface as a first proxy for biological membranes. While a monolayer can behave differently to a bilayer as the flexibility and the hydrophobicity of the air differs from that of the headgroups of the lipid, this approach minimizes substrate interactions ${ }^{21}$ associated with supported lipid bilayers, and is more straightforward to increase complexity of the system in terms of mixtures of phospholipids as well as additional components such as cholesterol and sphingomyelin in future studies. We have used the surface specific laser technique vibrational sum frequency spectroscopy (VSFS) to investigate propofol-induced changes in the molecular order in both the hydrophilic head group region and the hydrophobic tail region of three saturated phosphatidylcholines of different tail lengths $\left(\mathrm{C}_{14}, \mathrm{C}_{16}\right.$, and $\left.\mathrm{C}_{18}\right)$. Moreover, the propofol itself has been monitored in addition to the water molecules hydrating the lipids and the propofol. The studies have been performed at a surface pressure of $22-25 \mathrm{mN} \mathrm{m}^{-1}$, to mimic a biological membrane $\mathrm{e}^{22}$, as well as at $5 \mathrm{mN} \mathrm{m}^{-1}$, in order to gain understanding of how the surface pressure affects the ability of propofol to interact with phospholipids. To learn about the specific location of propofol in a phospholipid monolayer we also performed measurements on the $\mathrm{C}_{16}$-phospholipid using neutron reflectometry (NR) to resolve the location of the drug in the model monolayer.

\section{Experimental and Theoretical Methods}

\subsection{Materials}

Propofol (European Pharmacopoeia) was bought from Sigma Aldrich with a purity higher than 99\%. 1,2-distearoyl-sn-glycero-3-phosphocholine (DSPC), 1,2-dipalmitoyl-sn-glycero-3phosphocholine (DPPC), 1,2-dimyristoyl-sn-glycero-3-phosphocholine (DMPC), 1,2-distearoyl- 
d70-sn-glycero-3-phosphocholine-1,1,2,2-d4-N,N,N-trimethyl-d9 d-DSPC, 1,2-dipalmitoyl-d ${ }_{62}-$ $s n$-glycero-3-phosphocholine-1,1,2,2-d4-N,N,N-trimethyl-d9 d-DPPC, 1,2-dipalmitoyl-d $\mathrm{d}_{62}$-snglycero-3-phosphocholine $\mathrm{d}_{62}$-DPPC, and 1,2-dimyristoyl-d54-sn-glycero-3-phosphocholine1,1,2,2-d4-N,N,N-trimethyl-d9 d-DMPC were purchased from Avanti Polar Lipids with purity higher than 99\%, and were used as received. Their respective chemical structures are seen in Figure 1.

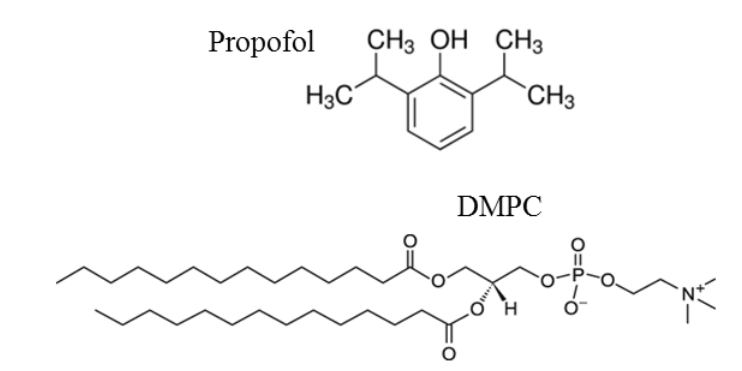

d-DSPC

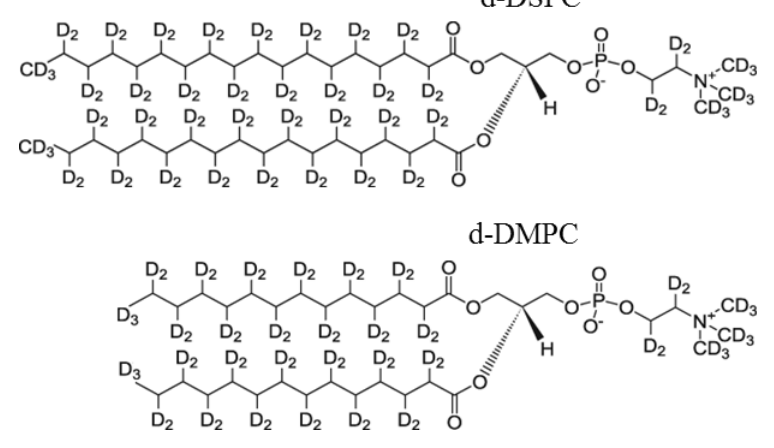

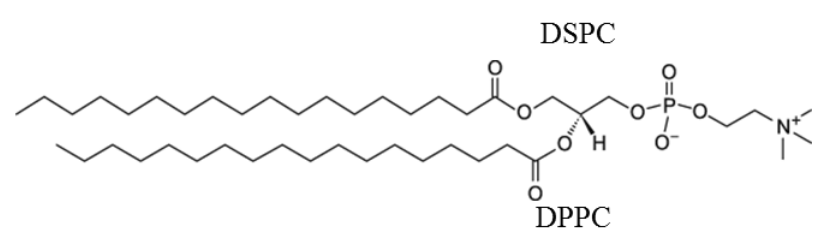

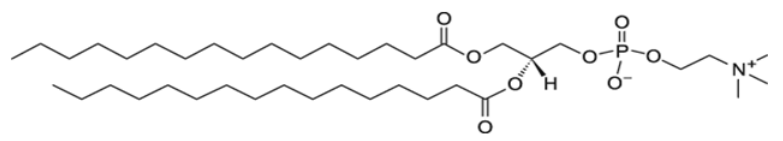

d-DPPC
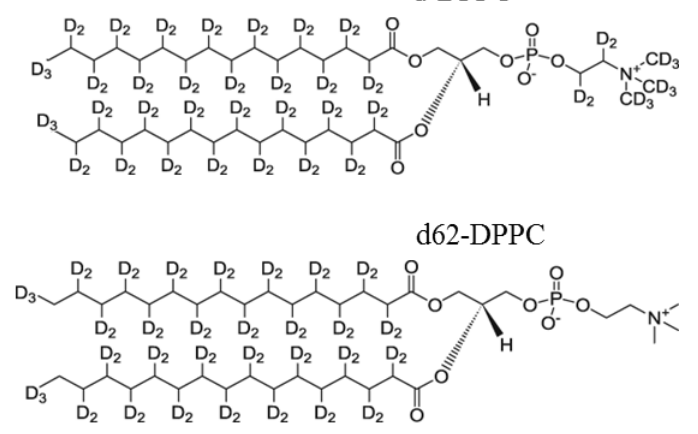

Figure 1. The chemical structures of the studied phospholipids and propofol.

Biological cell membranes contain both well-ordered and more fluid domains, and three lipids have been studied in order to elucidate the effect of propofol on both ordered and disordered monolayers, with DSPC $\left(\mathrm{C}_{18}\right)$ forming highly ordered structures, while DMPC $\left(\mathrm{C}_{14}\right)$ forms disordered layers. DPPC $\left(\mathrm{C}_{16}\right)$ is in between the two. The short tail saturated phospholipid DMPC was used rather than phospholipids with unsaturated fatty acid chains (which are common in cell membranes) to avoid oxidation of the double bond in contact with air, as it has 
been noticed in our previous work ${ }^{23-24}$. However, the short chain DMPC still possesses a highly disordered structure, similarly to the unsaturated phospholipids (e.g. POPC) in cell membranes, and serves, thus, as a good substitute.

\subsection{Experimental Protocols}

In order to investigate phospholipid monolayers at various surface pressures a KSV MiniMicro Langmuir trough made of Teflon $^{\mathrm{TM}}$ (with Derlin ${ }^{\circledR}$ hydrophilic barriers) was used in all experiments except NR, in which a Nima trough was used. Spreading solutions were prepared using chloroform (>99.8\%, Sigma Aldrich), and at least 5 min were allowed for chloroform evaporation before the experiments started. After spreading the monolayer either on pure water or $0.89 \mathrm{mM}$ propofol solution, the Delrin (polyoxymethylene) barriers were compressed at a constant rate of $6 \mathrm{~mm} / \mathrm{min}$. Compression measurements for DSPC, d-DSPC, DPPC, and d-DPPC were carried out at $21^{\circ} \mathrm{C}$, while for DMPC and d-DMPC the temperature was $26^{\circ} \mathrm{C}$ to ensure that the experiments were performed above the transition temperature occurring slightly above $20^{\circ} \mathrm{C}^{25,26}$. The surface pressures reported for mixed propofol/phospholipid systems are relative to a surface pressure of zero for the pure propofol solution, which itself has a surface pressure of $15 \mathrm{mN} \mathrm{m}^{-1}$. The concentration of $0.89 \mathrm{mM}$ propofol was chosen because the slope of the surface tension isotherm is constant, which indicates maximum surface coverage, yet the plateau has not been reached thus solubility issues resulting in beads of propofol sitting on the liquid surface are circumvented.

\subsection{Vibrational Sum Frequency spectroscopy (VSFS)}

Our VSFS instrument has been described in detail before ${ }^{24,27}$, and only a brief description is provided here. A Nd:YAG laser (PL-2251A-20, Ekspla) generates a $1064 \mathrm{~nm}$ wavelength beam at $20 \mathrm{~Hz}$, which is sent to an optical parametric generator/optical parametric amplifier (OPG/OPA 
- LaserVision). Here two beams are generated: a visible beam at a fixed wavelength of $532 \mathrm{~nm}$ and an IR beam tunable over the wavenumber region $1000-4000 \mathrm{~cm}^{-1}$ (approx. 20-400 $\mu \mathrm{J}$ ). The beams are subsequently overlapped on the sample surface (approx. $1 \mathrm{~mm}$ in diameter) to produce the sum frequency (SF) beam bearing the signature of the interfacial molecules. The SF beam passes through a monochromator and detected by a photomultiplier tube before the signal is processed using a computer where the SF intensity is normalized to the product of the intensities of the incoming visible and IR beams ${ }^{28}$ in order to account for beam intensity fluctuations.

The recorded sum frequency intensity, $I_{\mathrm{VSF}}$, is proportional to the square of the molecular number density multiplied with the orientationally averaged molecular hyperpolarizability $<\beta^{(2)}>$ of the probed moieties. For a molecule to be VSFS active it has to be both IR and Raman active, according to equation 1 :

$$
\beta_{a b c}^{(2)}=\frac{\alpha_{a b} \mu_{c}}{\omega_{n}-\omega_{\mathrm{IR}}-i \Gamma_{n}}
$$

where $a, b$ and $c$ are the molecular coordinates, $\alpha_{a b}$ the Raman polarizability tensor, $\mu_{c}$ the transition IR dipole moment, $\omega_{n}$ the peak position frequency, $\omega_{\mathrm{IR}}$ the irradiating infrared frequency, $\Gamma_{n}$ the damping constant of the $n^{\text {th }}$ resonant mode and $i$ the imaginary unit.

In order to access orientational information about the probed molecular species spectra with different polarizations of the three beams are needed, where $S$ and $P$ denote the polarizations perpendicular to and parallel with the plane of incidence, respectively. In the present case the spectra were acquired in the SSP polarization combination, where the repeat letters refer to the polarization of the SF, visible and IR beams, respectively.

\subsection{Neutron Reflectometry (NR)}

NR measurements were performed using the horizontal neutron reflectometer FIGARO at the Institut Laue-Langevin (Grenoble, France) ${ }^{29}{ }^{30}$. The technique provides a measure of the ratio of 
the number of neutrons in the specular reflection to those in the incident beam with respect to the momentum transfer, $q_{\mathrm{z}}$,

$$
q_{z}=\frac{4 \pi \sin \theta}{\lambda}
$$

where $\theta$ is the angle of incidence and $\lambda$ is the neutron wavelength ${ }^{31}$. Data were recorded at $\theta=$ $0.62^{\circ}$ and $3.78^{\circ}$ with chopper pulses of $7 \% \delta \lambda / \lambda$, and wavelengths with a range of $3-22 \AA$ were used to generate the reduced data. The background was subtracted from these data as a result of use of the area detector. The data analysis was carried out using Motofit ${ }^{32}$. More information about the modeling approach and the parameters used is described in the Supporting Information, and an extended description of the application of the technique to systems at fluid interfaces can be found in a recent review of the subject area ${ }^{30}$.

The interfacial structure of the adsorbed molecules at the air-water interface was resolved by comparing different structural models to data recorded in 4 isotopic contrasts: $\mathrm{d}_{62}-\mathrm{DPPC}_{2} \mathrm{D}_{2} \mathrm{O}$, $\mathrm{d}_{62}$-DPPC/ACMW, DPPC/D $\mathrm{D}_{2} \mathrm{O}$ and DPPC/ACMW, as in the approach used in ref. ${ }^{33}$, where ACMW is air contrast matched water, i.e. $8.1 \% \mathrm{v} / \mathrm{v} \mathrm{D}_{2} \mathrm{O}$ in $\mathrm{H}_{2} \mathrm{O}$. Data were recorded first on pure lipid monolayers at $5 \mathrm{mN} \mathrm{m}^{-1}$, and were then recorded on lipid monolayers spread on 0.89 $\mathrm{mM}$ propofol solution until the surface pressure was raised by $5 \mathrm{mN} \mathrm{m}^{-1}$.

The pure lipid monolayers were modeled using 2 interfacial layers: alkyl tails with a volume fraction of 1 , and hydrated head groups with a volume fraction calculated in such a way as to preserve an equal number of tails and head groups. Inter-layer roughness values of $3.0 \AA$ from capillary waves were included in the model. The monolayers with propofol were modeled by comparing 3 different locations of the drug: in the same layer as the lipid alkyl tails, in the same layer as the lipid head groups, and in a third layer under the lipid head groups. These 3 models were selected as being physically realistic possibilities as a result of the different driving forces 
associated with the interaction. More information about the modeling approach and the parameters used is described in the Supporting Information.

\section{Results and discussion}

\subsection{Surface pressure isotherm experiments}

The surface pressure isotherms for DSPC, DPPC, and DMPC monolayers on an aqueous subphase in the absence and presence of $0.89 \mathrm{mM}$ propofol are shown in Figure 2. The recorded surface pressure isotherms of these phospholipids in the absence of propofol are in agreement with data reported in the literature ${ }^{34-37}$. Briefly, upon compression DSPC goes directly from the so-called gaseous phase, where patches of ordered molecules are present in the monolayer ${ }^{24}$, to a condensed phase where the molecules are packed closely together over the entire surface.

On the contrary, the surface pressure isotherm for DPPC shows the presence of a liquid expanded phase, before a transition to a mixed liquid expanded/liquid condensed co-existence, and then a condensed phase at high surface pressures. Furthermore, the surface pressure isotherm for DMPC, due to its shorter hydrocarbon tail, does not show any condensed phase as the monolayer collapses directly from the liquid expanded phase at approximately $40 \mathrm{mN} \mathrm{m}^{-1}$.In the presence of propofol the mean molecular area for a given low surface pressure is larger for all three phospholipids. This is a clear indication that there is an interaction between propofol and the phospholipid monolayer. The isotherm of DSPC is strongly expanded at low surface pressures. The lift off point is shifted from about $57 \AA^{2}$. On water subphase containing $0.89 \mathrm{mM}$ propofol the surface pressure starts rising at about $130 \AA^{2}$.

The surface pressure increases gradually upon compression until about $10 \mathrm{mN} \mathrm{m}^{-1}$, at which point a condensed phase is achieved. Under this condition the area/molecule is similar to that found in the absence of propofol. 


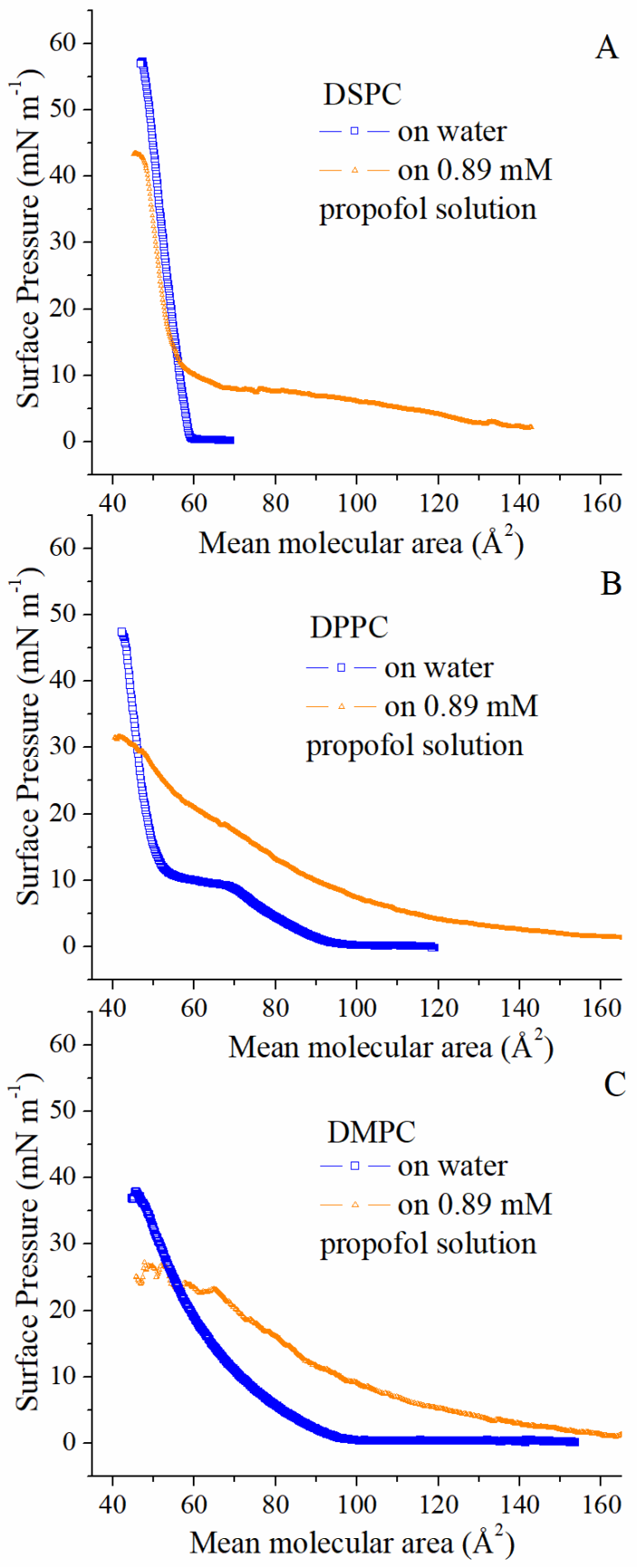

Figure 2. Comparison of A) DSPC, B) DPPC and C) DMPC surface pressure isotherms on a pure water subphase and on a $0.89 \mathrm{mM}$ propofol solution. The surface pressure is defined as the surface tension decrease compared to that of the respective subphase prior to spreading the phospholipid, i.e., without propofol pure water defines the surface pressure $0 \mathrm{mN} \mathrm{m}^{-1}$ and with propofol the pure propofol solution defines $0 \mathrm{mN} \mathrm{m}^{-1}$. 
This is the case due to the steep nature of the isotherm in the condensed phase, despite the different definition of zero pressure in the two experiments. This is a strong indication that propofol is removed from the interface upon compression.

In the case of DPPC and DMPC, the presence of propofol in the subphase also leads to an expansion of the monolayer at low surface pressures and a continuous increase in surface pressure without clear phase transitions. Therefore we may infer that the drug interactions invoke a fluidizing effect on the morphology of the monolayer.

All three systems (DSPC, DPPC, and DMPC) collapse at the same absolute surface pressure on both water and propofol solution, respectively. The apparent differences observed in Figure 2 are caused by the difference in surface tension reading before the experiment: $72 \mathrm{mN} \mathrm{m}^{-1}$ for pure water and $57 \mathrm{mN} \mathrm{m}^{-1}$ for propofol solution (which by our definition are both zero surface pressure). More precisely, at high areas per lipid molecule the relevant reference state is the propofol solution and this reference state is utilized in the figures. We note that the surface pressure of $0.89 \mathrm{mM}$ propofol solution relative to pure water is about $15 \mathrm{mN} \mathrm{m}^{-1}$. The collapse pressure of the phospholipids on the propofol solution is lower than that observed for the phospholipids relative to water. However, if we should compare these values against the same reference (pure water) we need to add $15 \mathrm{mN} \mathrm{m}^{-1}$ to the collapse pressure obtained on $0.89 \mathrm{mM}$ propofol solution. If this is done, the collapse pressure and thus the stability is the same on the pure water and the $0.89 \mathrm{mM}$ propofol subphase. This comparison is appropriate since, as we show below, propofol completely leaves the interface at high surface pressures. Therefore, we conclude that the stability of the monolayer is not significantly influenced by the presence of propofol in the subphase. 
It is interesting to note that qualitatively the changes to the surface pressure isotherms are reduced at higher surface pressures, which may mean that the drug is squeezed out of the monolayer with increasing surface pressure. However, a more direct probe of the interface such as VSFS is required in order to confirm this inference. In the following, directly connected to the isotherms, the vibrational features of the system involving water, propofol and phospholipid will be explored using VSFS, focusing mainly on propofol-induced changes in the terminal methyl, tail and head group regions of the phospholipids as well as on the water of hydration.

3.2 VSFS experiments: terminal methyl groups of propofol

In order to be able to observe propofol at different surface pressures from the VSF spectra, we have used phospholipids with deuterated tails and partially deuterated head groups to form monolayers on top of the $0.89 \mathrm{mM}$ propofol solution. Thus, the symmetric stretch of propofol at $2873 \mathrm{~cm}^{-1}$ is clearly distinguished from the phospholipid CD stretches and $\mathrm{CH}$ peaks in the region $2900 \mathrm{~cm}^{-1}$ to $2980 \mathrm{~cm}^{-1}$, originating from non-deuterated parts of the head group ${ }^{38}$ (see Figure 1 and Figure 3D).

Propofol, with a preferred orientational order, is present at the interface at low surface pressures for all three lipid systems. This is shown by two prominent peaks: the symmetric methyl stretch $\left(\mathrm{sCH}_{3}\right)$ stretch at $2873 \mathrm{~cm}^{-1}$ and the asymmetric $\mathrm{CH}_{3}\left(\mathrm{aCH}_{3}\right)$ at $2965 \mathrm{~cm}^{-1},{ }^{19}, 39$ (see Figure 4A). In the following we focus on the $\mathrm{sCH}_{3}$ around $2870 \mathrm{~cm}^{-1}$, since the $\mathrm{aCH}_{3}$ stretch at $2965 \mathrm{~cm}^{-1}$ overlaps with the non-deuterated $\mathrm{CH}_{2}$ peak at around $2950 \mathrm{~cm}^{-1}$ from the phospholipid. Further, the aromatic $\mathrm{CH}$ stretches of propofol appear above $3000 \mathrm{~cm}^{-1}$. Note that the VSF spectrum of propofol on water is shown in the inset in Figure 4A. Unfortunately, the spectra at low propofol concentrations have low intensities and we decided not to fit any of these spectra. 



Figure 3. Drop of the VSFS intensity of the $\mathrm{sCH}_{3}$ peak of propofol as a function of phospholipid surface pressure. $\mathrm{A}-\mathrm{dDSPC}, \mathrm{B}-\mathrm{dDPPC}, \mathrm{C}-\mathrm{dDMPC}$ all on propofol solution and $\mathrm{D}-\mathrm{dDSPC}$ on pure water.

To study how propofol penetrates the lipid monolayers, SSP spectra covering the propofol peak at about $2870 \mathrm{~cm}^{-1}$ were acquired at different surfaces pressures for all three deuterated lipids. As soon as the surface pressure starts to increase, and, accordingly, the mean lipid molecular area decreases, the symmetric $\mathrm{CH}_{3}$ signature of propofol at $2870 \mathrm{~cm}^{-1}$ decreases in all three cases, demonstrating the squeeze out of propofol from the monolayer with increasing surface pressure. For all lipids, it is obvious that the propofol signal decreases with increasing surface pressure over the whole range of surface pressures studied, displayed for 5, 13, and 24 
(22 for d-DMPC) $\mathrm{mN} \mathrm{m}^{-1}$ in Figure 3, where also the spectrum of a propofol solution without lipid is shown as reference in panels $\mathrm{A}-\mathrm{C}$. It is interesting to compare such behavior to that of a short designed antimicrobial peptide interacting with the phospholipids DPPC and 1,2dipalmitoyl-sn-glycero-3-phosphoglycerol, where in both cases the strength of the interaction was enhanced rather than diminished with increasing surface pressure ${ }^{40}$.

At the highest surface pressure (lowest area per lipid molecule), when the monolayers become tightly packed the symmetric $\mathrm{CH}_{3}$ signature of propofol disappears completely in the case of DSPC, and it becomes barely detectable in the monolayers formed by DPPC, while it is slightly more pronounced in the presence of DMPC. Thus, it appears that for the more disordered DMPC (see Figure 4) more propofol remains in the lipid region at higher surface pressures, presumably due to both its shorter alkyl chain and the difference in head group hydration ability. We will discuss this argument later in this paper.

The concentration of the propofol in the interfacial region is gradually changing with the surface pressure of the monolayer. Since the VSFS response depends on both the amount and the average orientation of this species the quantification of the interfacial propofol becomes impractical.

\subsection{VSFS experiments: phospholipid tails}

The VSFS technique is sensitive to conformational changes in alkyl tails present at an interface $^{24,41}$. The order in the monolayer can be estimated from the intensity ratio of the symmetric methyl stretch $\left(2875 \mathrm{~cm}^{-1}\right)$ to the symmetric methylene stretch $\left(2850 \mathrm{~cm}^{-1}\right)^{42,43}$. Generally, when a monolayer is in an ordered state the backbone bonds adopt an all-trans configuration, and the methylene groups generate little or no signal since there is an inversion center between each pair of methylene groups. As the monolayer turns into a more liquid-like or 
disordered state, gauche defects start to occur, breaking the symmetry, which increases the methylene signal. The methyl group will always generate a signal since it resides in a noncentrosymmetric environment (hydrocarbon tails below and air above). Thus, a high $\mathrm{CH}_{3} / \mathrm{CH}_{2}$ intensity ratio signifies an ordered tail region, whereas a low ratio is observed in disordered systems. Due to the fact that the recorded $\mathrm{CH}$ stretches of the phospholipid tail have some contribution from the propofol $\mathrm{CH}$-groups we have chosen not to fit the spectra. The overlap between the two stretches is visible in both Figure 4 and Figure 6 .

$D S P C$

The alkyl tails of DSPC are ordered at high surface pressure both on pure water ${ }^{44}$ and on the aqueous propofol solution. This is clearly shown in Figure 4B by the dominance of $\mathrm{sCH}_{3}$ (2 875 $\left.\mathrm{cm}^{-1}\right)$ and its Fermi resonance $\mathrm{sCH}_{3} \mathrm{FR}\left(2937 \mathrm{~cm}^{-1}\right)$ over the significantly less intense symmetric methylene stretch $\mathrm{sCH}_{2}\left(2850 \mathrm{~cm}^{-1}\right)$. The two spectra recorded on the two subphases are in fact very similar. This is consistent with the data shown in Figure 3, suggesting that essentially no propofol is present in the interfacial region at high compression, in accordance with the Langmuir isotherms in Figure 2A, where the curves below molecular areas $\sim 55 \AA^{2}$ essentially overlap.

At lower surface pressure $\left(5 \mathrm{mN} \mathrm{m}^{-1}\right)$ on the water subphase the intensity of the $\mathrm{sCH}_{3}$ still dominates over the weak $\mathrm{sCH}_{2}$, showing that the alkyl tails remain in a highly ordered state. However, when propofol is present in the subphase, the monolayer is more expanded at $5 \mathrm{mN} \mathrm{m}^{-}$ ${ }^{1}$ and the $\mathrm{sCH}_{2}$ peak intensity (at $\sim 250 \mathrm{~cm}^{-1}$ ) becomes dominant. Thus, the presence of propofol induces disorder in the phospholipid tail region at low surface pressures. 

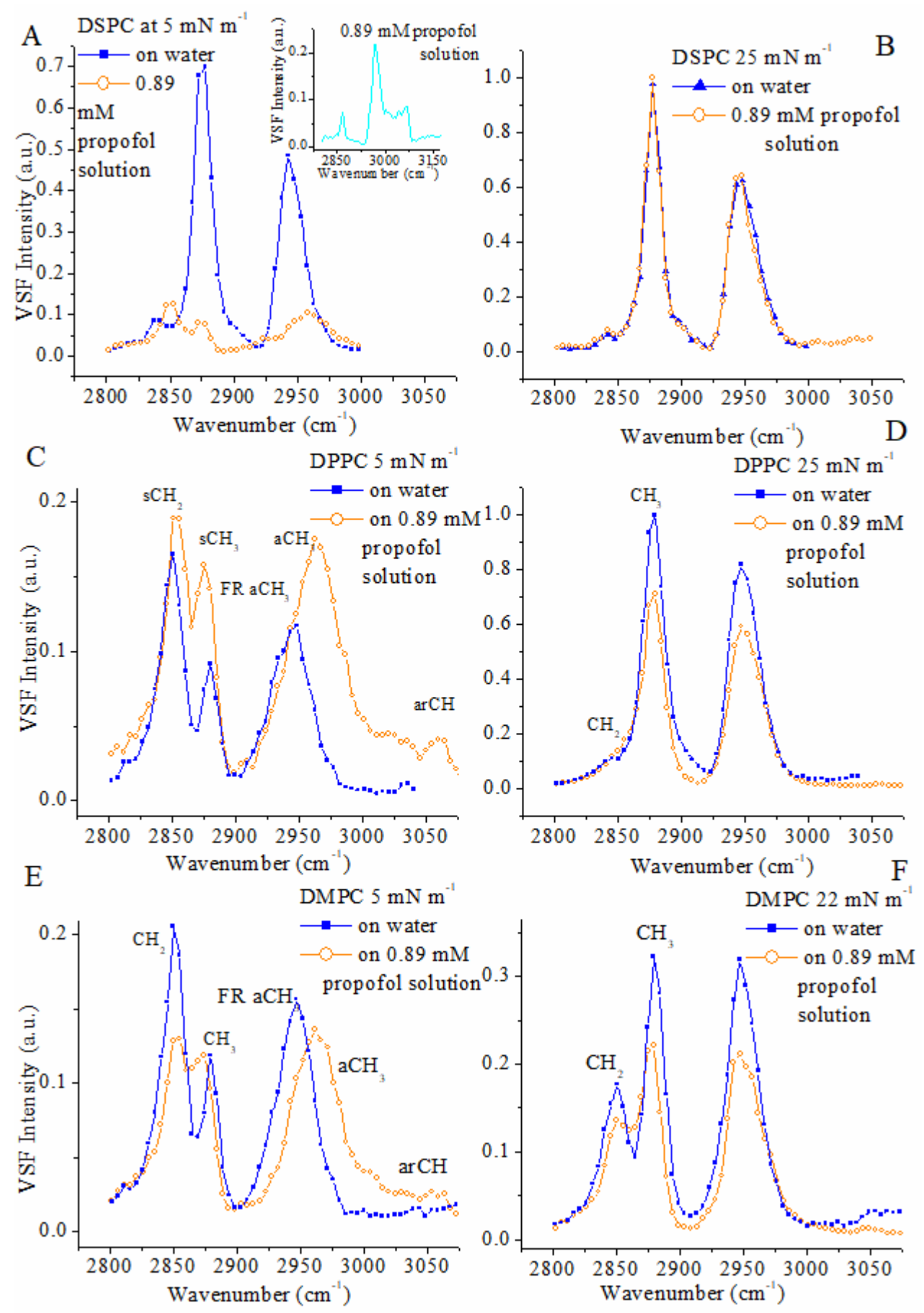

Figure 4. Comparison of VSF spectra of the three phospholipids (DSPC, DPPC, and DMPC) in the $\mathrm{CH}$ region at $5 \mathrm{mN} \mathrm{m}^{-1}$ and $25 \mathrm{mN} \mathrm{m}^{-1}\left(22 \mathrm{mN} \mathrm{m}^{-1}\right.$ for DMPC) in the absence and presence of $0.89 \mathrm{mM}$ propofol in the subphase. All peaks are normalized to the intensity of the $\mathrm{sCH}_{3}$ of DSPC at $25 \mathrm{mN} \mathrm{m}^{-1}$, and their absolute intensities can be directly compared in all plots. 
Additionally, a peak at $2965 \mathrm{~cm}^{-1}$ becomes more distinct, and this peak likely carries contributions from both the antisymmetric methyl stretch of propofol and DSPC, although we believe that propofol is responsible for the main part due to the appearance of the band upon addition of propofol.

$D P P C$

The tail region of the DPPC monolayer at high surface pressure $\left(25 \mathrm{mN} \mathrm{m}^{-1}\right)$ is in an ordered state both in the absence and presence of propofol (Figure 4D). This is shown by the predominance of the $\mathrm{sCH}_{3}$ at $2875 \mathrm{~cm}^{-1}$ over the very weak symmetric methylene stretch at $\sim 2$ $850 \mathrm{~cm}^{-1}$. The overall peak intensities are slightly weaker in the presence of propofol. This can be explained by a slightly decreased number of probed molecules due to the increased mean molecular area, as seen in Figure 2.

At low surface pressure $\left(5 \mathrm{mN} \mathrm{m}^{-1}\right)$, the intensity of the $\mathrm{sCH}_{2}$ peak from DPPC is stronger than the intensity of the $\mathrm{sCH}_{3}$ peak both with and without propofol present in the subphase, demonstrating significant disorder in the tail region. Even though the number of $\mathrm{CH}_{2}$ oscillators probed in the expanded state in the presence of propofol is significantly lower $(\sim 30 \%)$ compared to on the pure water subphase, the intensity of the $\mathrm{CH}_{2}$ peak is stronger due to more gauche defects induced by the interaction with propofol. The presence of ordered propofol in the interfacial region at this low surface pressure is demonstrated by the $\mathrm{aCH}_{3}$ at $2965 \mathrm{~cm}^{-1}$ and the aromatic $\mathrm{CH}$ peaks at around $3030 \mathrm{~cm}^{-1}$ and $3071 \mathrm{~cm}^{-1}$, respectively, which also are seen in Figure 6, although the peak at $2965 \mathrm{~cm}^{-1}$ probably also carries contributions from the lipid. Additionally, the propofol $\mathrm{sCH}_{3}$ feature contributes to the overall intensity of the $2875 \mathrm{~cm}^{-1}$ peak, even though it is difficult to separate it from the $\mathrm{sCH}_{3}$ emanating from the phospholipid. 


\section{$D M P C$}

The hydrocarbon tails of DMPC (Figure 4E and 4F) are, in contrast to those of DSPC and DPPC, in a low ordered state even at high surface pressure $\left(22 \mathrm{mN} \mathrm{m}^{-1}\right)$, both in the absence and presence of propofol in the subphase. This is demonstrated by the methylene peak at $2850 \mathrm{~cm}^{-1}$, which becomes comparable in intensity to that of the methyl peak at $2875 \mathrm{~cm}^{-1}$. In both Figure $4 \mathrm{E}$ and $\mathrm{F}$ in the presence of propofol the apparently less resolved peaks in the region around 2 $850-2875 \mathrm{~cm}^{-1}$ originate from the contribution of the symmetric methyl stretch of both DMPC and propofol. The spectra with and without propofol at $22 \mathrm{mN} \mathrm{m}^{-1}$ are quite similar, although the spectrum recorded in the presence of propofol is weaker due to the larger area per molecule and increased tail disorder. Further, the presence of propofol is revealed by the weak shoulder at 2 $965 \mathrm{~cm}^{-1}$, which in the case of $5 \mathrm{mN} \mathrm{m}^{-1}$ dominates over the Fermi resonance at $2937 \mathrm{~cm}^{-1}$. At both surface pressures ordered propofol is also observed at the interface through its aromatic $\mathrm{CH}$ $\left(3071 \mathrm{~cm}^{-1}\right)$ peak.

\section{Summary}

Our data show that propofol to a large extent leaves the interfacial region as the phospholipid monolayers are compressed, and, therefore, its presence in the subphase has less effect on the tail packing and order at high surface pressure $\left(22-25 \mathrm{mN} \mathrm{m}^{-1}\right)$ for the three phosphatidylcholines investigated. This mode of interaction contrasts with that of a recently-studied peptide which enhanced its interaction with both zwitterionic and anionic phospholipid monolayers with increasing surface pressure. At low surface pressure $\left(5 \mathrm{mN} \mathrm{m}^{-1}\right)$, on the other hand, the tail region of all three phospholipids is significantly more disordered in the presence of propofol. It thus appears that propofol promotes disorder, which is consistent with computer simulations 
suggesting preferential accumulation of small hydrophobic and amphiphilic molecules at the border between ordered and disordered domains or inside the disordered domains ${ }^{45,46}$.

\subsection{VSFS experiments: phospholipid headgroups}

Above it was shown that phospholipids with the same headgroup but with different length of the hydrocarbon tail respond differently when in contact with propofol. The focus will now be towards the behavior of the headgroup, which is the same for all three phospholipids (Figure 1). The SSP spectra of the phospholipids in the $\mathrm{PO}$ and $\mathrm{C}=\mathrm{O}$ regions are shown in Figure 5. The spectra recorded in the absence and presence of $0.89 \mathrm{mM}$ propofol are compared at low $(5 \mathrm{mN}$ $\mathrm{m}^{-1}$ ) and high (22 or $25 \mathrm{mN} \mathrm{m}^{-1}$ ) surface pressure. The most prominent peak is the symmetric $\mathrm{PO}_{2}$ stretch, which is centered at $1090 \mathrm{~cm}^{-1}$ with a shoulder summing the C-OP and CO-O-C stretches at $1050 \mathrm{~cm}^{-1}$ and $1075 \mathrm{~cm}^{-1}$, respectively ${ }^{38}$.

The other three peaks are the antisymmetric $\mathrm{PO}_{2}$ stretch, the deformation $\mathrm{CH}_{3}$, and the $\mathrm{C}=\mathrm{O}$ stretch, which are barely visible at $1250 \mathrm{~cm}^{-1}, 1460 \mathrm{~cm}^{-1}$, and $1750 \mathrm{~cm}^{-1}$, respectively ${ }^{38}$. Thus, here we focus on the symmetric $\mathrm{PO}_{2}$ peak, since it is most intense, and how it is affected by propofol. No signal was observed in the PPP polarization combination in any spectrum, and the acquisition of SPS spectra was not possible since our half-wave plate $\left(\mathrm{MgF}_{2}\right)$ is not transparent in the region around $1100 \mathrm{~cm}^{-1}$. Further, especially at $5 \mathrm{mN} \mathrm{m}^{-1}$, it is essentially impossible to distinguish the contributions from the various peaks in the region around the symmetric $\mathrm{PO}_{2}$ stretch. Hence, drawing conclusions about how the amplitudes change from fitting the spectra would be unreliable, and we only discuss the propofol induced spectral changes qualitatively. Due to the issues mentioned above it was not possible to study orientational changes of the headgroup. It has been shown that the $\mathrm{PO}_{2}$ peak position is sensitive to its environment. It can be 
shifted by $10-15 \mathrm{~cm}^{-1}$ due to counterion complexation, hydration state, and conformational changes $^{47-51}$.
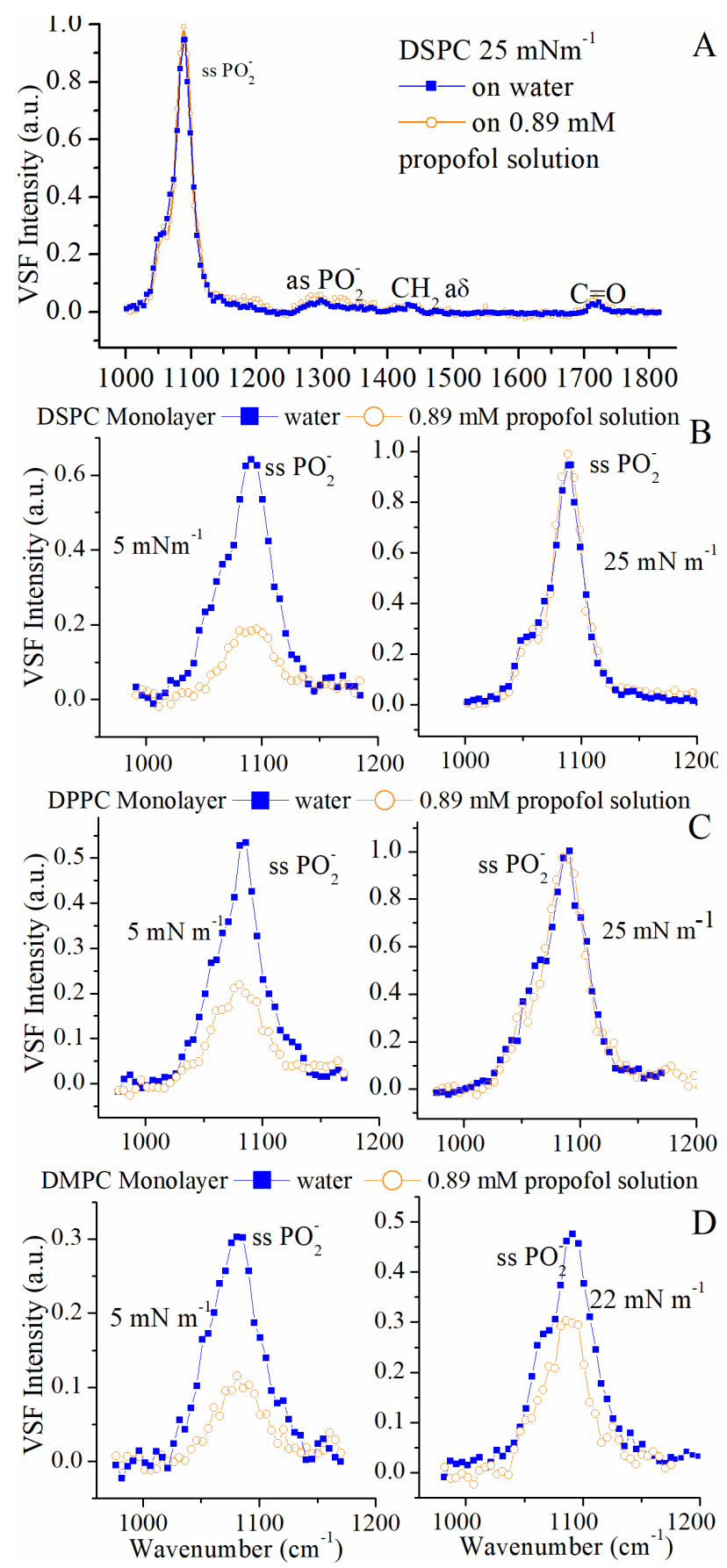

Figure 5. VSF spectra of the three phospholipids A) and B) DSPC, C) DPPC, and D) DMPC in the fingerprint region in the absence and presence of $0.89 \mathrm{mM}$ propofol in the subphase at two different surface pressures: 5 and $25 \mathrm{mN} \mathrm{m}^{-1}\left(22 \mathrm{mN} \mathrm{m}^{-1}\right.$ for DMPC). All features are 
normalized to the intensity of the $\mathrm{SPO}_{2}$ at $25 \mathrm{mN} \mathrm{m}^{-1}$ of DSPC hence the absolute intensities can be directly compared between different lipids.

However, the presence of propofol in the subphase does not result in any measurable shift of the $\mathrm{PO}_{2}$ peak position for any phospholipid at any surface pressure as observed from Figure 5. Hence, there is no interaction between the phosphate group and propofol that is strong enough to affect the $\mathrm{PO}_{2}$ vibration. The intensity of the symmetric $\mathrm{PO}_{2}$ stretch depends on the number density and the average orientation of the head group. Its magnitude will decrease when the order in the head group region decreases and when the average orientation of the $\mathrm{PO}_{2}$ dipole transition moment changes to become more parallel to the surface (since SSP probes dipole transitions along the surface normal), as well as when the surface number density is reduced.

\section{$D S P C$}

The spectral features of DSPC at high surface pressure $\left(25 \mathrm{mN} \mathrm{m}^{-1}\right)$ are the same in the presence and absence of propofol, as seen in Figure 5A and B. This fact, as well as the spectra recorded in the tail region (Figure 3), demonstrates that there is no significant perturbation of the DSPC monolayer induced by propofol (neither the tails nor the headgroup). This result is consistent with the complete squeeze out of propofol from DSPC monolayers at high surface pressures, as discussed above. At $5 \mathrm{mN} \mathrm{m}^{-1}$ (Figure 5B) it is seen that the $\mathrm{PO}_{2}$ intensity is significantly weaker in the presence of propofol than without. This is similar to what is observed for the $\mathrm{CH}$ stretching region in Figure 4. The reduction of order in the tails is obvious in Figure 4 from the reduced $\mathrm{CH}_{3} / \mathrm{CH}_{2}$ intensity ratio, but a reduced order in the headgroup cannot be inferred from the spectra since no peak shifts are observed, and the reduction in signal to a large extent depends on the reduced DSPC number density in comparison with the case of pure water 
(Figure 2). Hence, we conclude that the main origin of the reduction in peak intensity at $5 \mathrm{mN} \mathrm{m}^{-1}$ with propofol is due to a reduced DSPC number density at the water surface.

\section{DPPC}

Just as for DSPC, the presence of propofol in the subphase does not affect the spectra for DPPC in the head group region at a surface pressure of $25 \mathrm{mN} \mathrm{m}^{-1}$ (Figure $5 \mathrm{C}$ ). This agrees to a large extent with the DPPC spectra in the $\mathrm{CH}$ region, although the intensity is slightly lower in the presence of propofol in the $\mathrm{CH}$ region. Moreover, the $\mathrm{PO}_{2}$ intensity for DPPC is as high as for DSPC, indicating a similar packing and orientation of the headgroups for these lipids, since the molecular area is almost similar (Figure 2). Thus, for both DSPC and DPPC we can draw the conclusion that propofol does not induce any significant changes in the phospholipid headgroup at a biological surface pressure of $25 \mathrm{mN} \mathrm{m}^{-1}$. The $\mathrm{PO}_{2}$ intensity is significantly lower in the presence compared with the absence of propofol at $5 \mathrm{mN} \mathrm{m}^{-1}$, but no clear peak shifts are observed. Based on the large difference in molecular area in Figure 2 with and without propofol at $5 \mathrm{mN} \mathrm{m}^{-1}$, we attribute the reduction in $\mathrm{PO}_{2}$ intensity in the presence of propofol mainly due to a reduced number density of DPPC, similar to DSPC.

\section{DMPC}

Already at a high surface pressure $\left(25 \mathrm{mN} \mathrm{m}^{-1}\right.$ for DSPC and DPPC, $22 \mathrm{mN} \mathrm{m}^{-1}$ for DMPC), the $\mathrm{PO}_{2}$ signal for DMPC (Figure 5D) spread on pure water is significantly lower (around 50\%) than for DPPC and DSPC. This is to some extent due to the higher molecular area for DMPC in comparison with the other lipids (see Figure 2). However, it could in addition be due to a different orientation and an increased disorder of the headgroup. This is due to the fact that DMPC exists in a liquid expanded state and the other lipids in a condensed state at these surface high pressures, meaning that the hydrocarbon chains in DMPC are significantly more disordered 
compared with DSPC and DPPC (Figure 3B, 3D and 3F). Addition of propofol induces therefore a reduction in peak intensity, which likely originates from a reduced lipid number density to the largest extent. Similarly, to DSPC and DPPC, the peak intensity is lower at $5 \mathrm{mN} \mathrm{m}^{-1}$ compared with $22 \mathrm{mN} \mathrm{m}^{-1}$, also a result of a reduced number density. A comparison of the intensity of the spectrum in the absence and presence of propofol at $5 \mathrm{mN} \mathrm{m}^{-1}$ shows that there is a notable reduction in intensity in the latter case, which follows given the large reduction in lipid number density (Figure 2).

\section{Summary}

The overall picture that emerges from the studies of the headgroup confirms the observation from the $\mathrm{CH}$ stretching region, that propofol is excluded from ordered phospholipid regions with increasing surface pressure and, thus, preferentially accumulates in disordered regions or at the border between ordered and disordered regions. This suggests that there is a non-homogeneous distribution of propofol in the lateral direction of phospholipid monolayers consisting of both ordered and disordered regions.

These conclusions appear to be largely consistent with computer simulations of small amphiphilic molecules interacting with phospholipid membranes ${ }^{45}, 46,52$. Suggesting that propofol in the normal direction preferentially resides at the border between the non-polar and polar region of the phospholipid monolayer, just as suggested in studies of DPPC and propofol ${ }^{17}$ and for ethanol by computer simulations ${ }^{52}$. Since VSFS does not provide irrefutable direct information about the location of the propofol in the lipid monolayer we will return to this issue later when discussing results from NR below.

The result that propofol affects disordered phospholipids to greater extent than when ordered is interesting in view of the fact that cell membranes consist of both ordered and disordered lipid 
domains. Hence, these studies show that propofol in the human body potentially interacts with and penetrates cell membranes in the disordered parts more than the highly ordered lipid rafts.

\subsection{VSFS experiments: water}

We turn our attention now to how the presence of propofol affects the water molecules residing next to the phospholipid monolayer, starting by briefly reviewing the interpretation of the VSF spectra of the pure water interface shown in Figure 6A. It is unambiguously agreed that the feature at $3700 \mathrm{~cm}^{-1}$ is the free $\mathrm{OH}$ bond that vibrates against air, and, as such, it does not participate in any hydrogen bonding. The assignment of the broad band from approximately 2 800 to $3600 \mathrm{~cm}^{-1}$, however, has been for long time been a source of debate ${ }^{53-60}$. Nevertheless, it is generally agreed that the hydrogen bond strength varies across the full width of this broad band, so that the $\mathrm{OH}$ signal is a signature of a collective vibration of several water molecules. VSF spectra of water hydrating phospholipid monolayers have been extensively studied ${ }^{61-63}$. Water molecules that are present in the proximity of the zwitterionic phospholipid head group of phosphatidylcholines are oriented by the electric field from these groups, and the orientation of the water molecule thus depends on its specific location with respect to the headgroup ${ }^{49,64}$.

Water of hydration will in the following be regarded as water in direct contact with the phospholipid headgroup together with the water oriented in the electric field. The latter contribution is considered to be dominant for a charged phospholipid ${ }^{49}$. A study of a DPPC monolayer on water employing phase sensitive VSFS revealed that the water molecules contributing to the spectra in the wavenumber range $3000-3550 \mathrm{~cm}^{-1}$ are preferentially oriented with their hydrogen atoms towards the monolayer ${ }^{49}$. Moreover, Mondal et al ${ }^{65}$ have shown that water species having opposite orientation coexist at the interface, the strongly hydrogen bonded water existing in the vicinity of the anionic moiety with net hydrogen up orientation and the 
weakly hydrogen bonded water existing in the vicinity of the cationic moiety with net hydrogen down orientation.
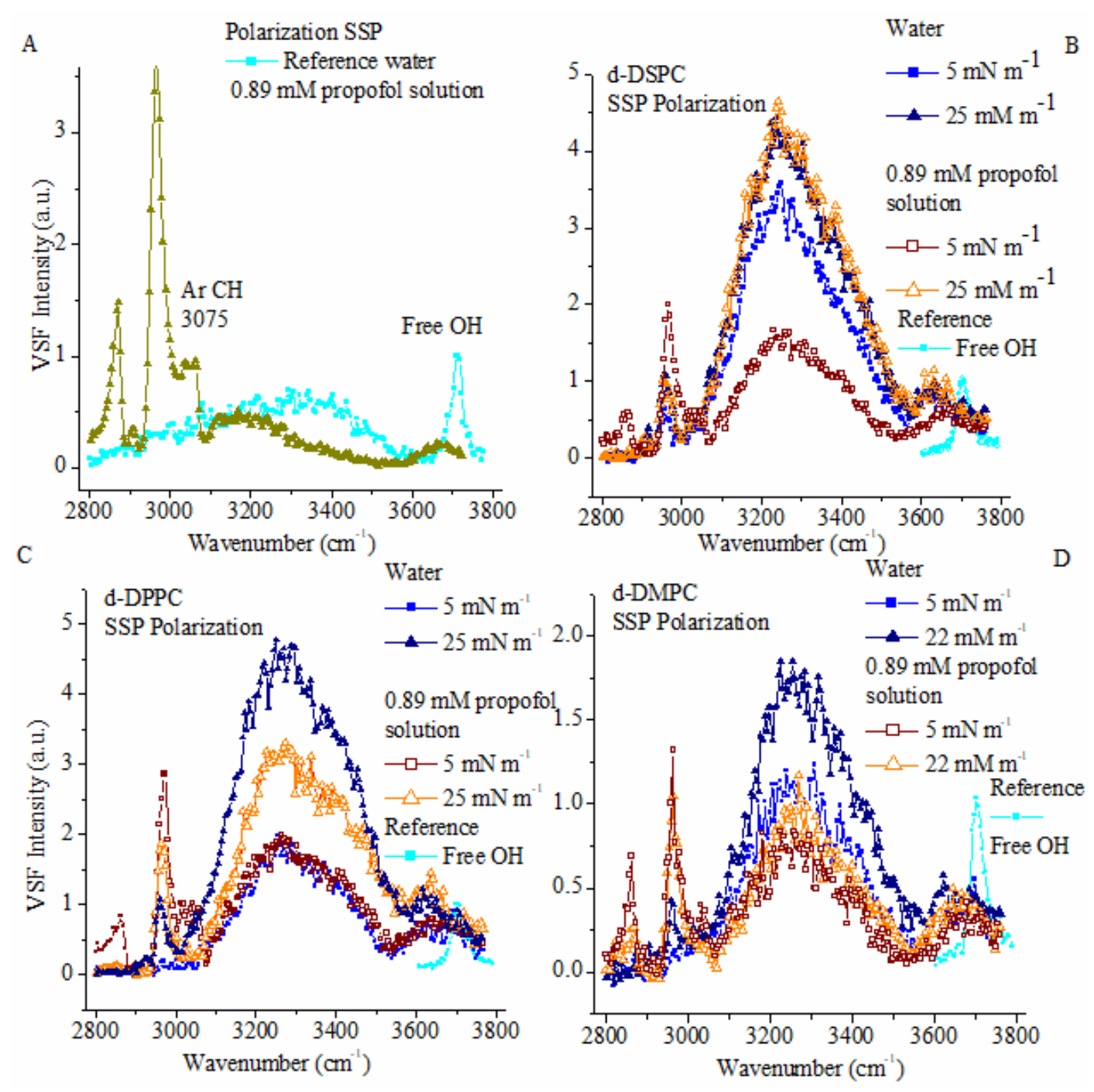

Figure 6. Water spectra of A) air-water interface in the absence and presence of $0.89 \mathrm{mM}$ propofol, B) d-DSPC C) d-DPPC, and D) d-DMPC phospholipid monolayers on pure water and on $0.89 \mathrm{mM}$ propofol solutions at selected surface pressures in SSP polarization.

In the present study we use the deuterated form of the phospholipids in order to reduce the spectral interference between the strong methyl peaks of the phospholipids and the weaker water 
bands, and to distinguish the propofol $\mathrm{sCH}_{3}$ peak at $2873 \mathrm{~cm}^{-1}$ from the corresponding peak of the lipids.

The VSF spectrum of the water surface in the presence of $0.89 \mathrm{mM}$ propofol is shown in Figure $6 \mathrm{~A}$ where the strength of the free $\mathrm{OH}$ vibration at $3700 \mathrm{~cm}^{-1}$ on pure water is a reference defining one arbitrary unit (a.u.). It is significantly different to that recorded in the absence of propofol. First, we notice a spectral feature centered at about $3150 \mathrm{~cm}^{-1}$ that is due partly to the $\mathrm{OH}$ group $^{58}$ of propofol and partly to strongly hydrogen bonded water interacting with the $\mathrm{OH}$ group of propofol present in the interfacial layer. Weakly hydrogen bonded water is also observed by one small band at about $3660 \mathrm{~cm}^{-1}$, which we assign to $\mathrm{O}-\mathrm{H}$ vibrations affected by water-hydrocarbon interactions ${ }^{66-68}$. Moreover, the water region of the VSF spectrum of the propofol solution shows that the free $\mathrm{OH}$ vibration at $3700 \mathrm{~cm}^{-1}$ has vanished, and hence no $\mathrm{OH}$ bonds protruding out into the air exist. The hydrocarbon peaks from propofol are observed in the low wavenumber region $\left(<3100 \mathrm{~cm}^{-1}\right)$.

\section{DSPC}

The water spectra in the presence of a d-DSPC monolayer on a pure water subphase (Figure $6 \mathrm{~B}$ ) show that the free $\mathrm{OH}$ vibration is absent at a surface pressure of $5 \mathrm{mN} \mathrm{m}^{-1}$, and instead the peak due to weakly interacting water molecules with the hydrocarbon tails $\left(3650 \mathrm{~cm}^{-1}\right)$ appears. This peak remains as the surface pressure is increased from $5 \mathrm{mN} \mathrm{m}^{-1}$ to $25 \mathrm{mN} \mathrm{m}^{-1}$. The intensity of the broad water band $\left(\sim 3000-3500 \mathrm{~cm}^{-1}\right)$ due to hydration of the phospholipid headgroup increases slightly with increasing surface pressure from $5 \mathrm{mN} \mathrm{m}^{-1}$ to $25 \mathrm{mN} \mathrm{m}^{-1}$ as a result of a higher surface density of headgroups, and this observation is consistent with earlier reports ${ }^{44}$. The overall shape of these adsorption bands is unaltered as the surface pressure is increased; indicating that the hydrogen bond strength distribution is unchanged. 
For DSPC monolayers spread on propofol solution at $5 \mathrm{mN} \mathrm{m}^{-1}$, the propofol is present at the interface as revealed by the $\mathrm{sCH}_{3}$ band at 2873 at $\mathrm{cm}^{-1}$ and the $\mathrm{aCH}_{3}$ band at $2965 \mathrm{~cm}^{-1}$ (overlapping with the adjacent d-DSPC band) that can be assigned to the drug. At this surface pressure, the intensity of the spectra of the water region is decreased in the presence of propofol in the subphase. One reason is that there are fewer phospholipid head groups per unit area (see Figure 2A) to induce the electric field that orients the water molecules. The other reason is that the presence of propofol at the interface reduces the number of water molecules in this region, since propofol aligns fewer water molecules than the lipid.

The spectra from the whole water region $\left(\sim 3000-3800 \mathrm{~cm}^{-1}\right)$ taken at a d-DSPC surface pressure of $25 \mathrm{mN} \mathrm{m}^{-1}$ overlap perfectly in the absence and the presence of propofol. Thus, the water residing at the interface shows a completely similar structure in the absence and presence of propofol in the bulk solution. Further, at this surface pressure the propofol $\mathrm{sCH}_{3}$ band at 2873 $\mathrm{cm}^{-1}$ has completely disappeared, and in the region around $2960 \mathrm{~cm}^{-1}$ the spectral features completely overlap (thus, there are only contributions from the d-DSPC band at $2956 \mathrm{~cm}^{-1}$ whereas the propofol band at $2965 \mathrm{~cm}^{-1}$ has vanished). All of these observations support the interpretations above that propofol has been fully squeezed out of the monolayer at the high surface pressure and none remains present at the interface.

We note that the maximum intensity of the broad $\mathrm{OH}$ band is approximately eight times lower on a subphase containing only propofol (no phospholipid), compared to the case with only dDSPC on pure water at $25 \mathrm{mN} \mathrm{m}^{-1}$ (Figures 6A and 6B have comparable scale). We infer that this mainly is a consequence of the stronger electrical field from the zwitterionic choline group of DSPC compared to that emanating from the dipolar and uncharged OH-group of propofol. This suggests that d-DSPC affects the probed water molecules more than propofol, and as a 
consequence the maximum intensity of the broad OH-band is observed at $\sim 3250 \mathrm{~cm}^{-1}$ for dDSPC both in the absence and presence of propofol, and not at $3150 \mathrm{~cm}^{-1}$ where the OH-band recorded on a propofol solution in the absence of phospholipids appears.

\section{DPPC}

The water spectra for the DPPC monolayer, shown in Figure 6C, have many similarities to those recorded for the DSPC monolayer. One difference is that, at $5 \mathrm{mN} \mathrm{m}^{-1}$ in the absence of propofol, the water band is weaker for the monolayer of DPPC than for that of DSPC. This is mainly due to the larger molecular area in the DPPC monolayer (see Figure 2).

At low surface pressure $\left(5 \mathrm{mN} \mathrm{m}^{-1}\right)$, where the DPPC monolayer is in a liquid expanded state, the broad water band is basically unaffected by the presence of propofol over its entire region, spanning the range from $\sim 3100 \mathrm{~cm}^{-1}$ to $3800 \mathrm{~cm}^{-1}$. This is the case in spite of the fact that both d-DPPC and propofol are present in the surface region as revealed by their characteristic peaks. Hence, the surface composition is different on the pure water and on the propofol solution, but the water bands still overlap. This is an intriguing fact that originates from several different parameters, such as the molecular orientation and surface density of propofol and DPPC. Since the lipid results in a stronger water signal than propofol and the lipid number density is lower at $5 \mathrm{mN} \mathrm{m}^{-1}$ (figure 2B) in the presence of propofol, there must be another parameter compensating this and hence results in similar band intensities. Such a parameter is the average orientation of the lipid. However, since the phosphate headgroup only was detected in the SSP polarization and the fact that the lipid and propofol bands overlap in figure 4C ( $\mathrm{CH}$ region), it is difficult to judge how pronounced the effect of orientation is in ordering water layers.

As the DPPC monolayer is compressed to a more ordered state, the VSFS signal of the broad water band increases due to the increased surface density of DPPC. At a surface pressure of 25 
$\mathrm{mN} \mathrm{m}^{-1}$, the water band is less intense in the presence of propofol in the subphase, and some propofol remains at the interface as evidenced by a trace of the propofol $\mathrm{aCH}_{3}$ band $\left(2965 \mathrm{~cm}^{-1}\right)$ overlapping the phospholipid non-deuterated $\mathrm{CH}_{2}$ band $\left(2956 \mathrm{~cm}^{-1}\right)$, as well as a barely visible peak at $2873 \mathrm{~cm}^{-1}$. We attribute the decreased water band intensity to a slightly reduced surface density (figure 2B), whereas the packing order essentially is the same as revealed by the similar spectra in figure $4 \mathrm{D}$.

\section{$D M P C$}

The water bands observed for the DMPC monolayer without propofol, Figure 6D, have obvious similarities to those of the other two phosphatidylcholines. However, the intensities are significantly weaker at both low and high surfaces pressures due to the lower phospholipid number density (Figure 2) and also due to the more disordered phospholipid structure (high $\mathrm{CH}_{2} / \mathrm{CH}_{3}$ intensity ratio in figure $4 \mathrm{E}$ and $\mathrm{F}$ ). In the absence of propofol the water band increases with increasing surface pressure as the surface density of DMPC increases, and moreover, the ordering of at least the chains increases, as seen in Figure 4E and F. The water band intensities are lower on the propofol-containing subphase, mainly due to the presence of propofol at the interface that reduces the surface density of DMPC, and, hence, the ordering of water molecules from the charged lipid headgroup. Clear peaks at $2873 \mathrm{~cm}^{-1}$ and $2965 \mathrm{~cm}^{-1}$ reveal the presence of propofol at both low and high surface pressure, where the partly squeezed out propofol is revealed by the lower peak intensities at the higher surface pressure.

\section{Summary}

The intensity of the water bands increases with surface density and phospholipid ordering. Moreover, the hydration water of DSPC, DPPC, and DMPC is affected differently by the presence of propofol, which, since the headgroup is the same, is attributed to the different 
propensity of the phospholipids to form ordered conformations, as judged from the VSF spectra of the tail region. Besides the fact that more disorder yields a lower signal, it also results in a lower lipid surface density for a given surface pressure, which further contributes to a reduction in signal. For DSPC, the water hydrating the ordered layer that is present on the water subphase is affected by propofol at low surface pressure $\left(5 \mathrm{mN} \mathrm{m}^{-1}\right)$, but not at high surface pressures when propofol is removed from the interfacial region. At $5 \mathrm{mN} / \mathrm{m}$ DPPC is fairly disordered both with and without the presence of propofol, a parameter which leads to a reduction in intensity of the water bands. The intensity of the water band in the presence of DPPC is reduced by propofol at $25 \mathrm{mN} \mathrm{m}^{-1}$ due to the presence of small amounts of propofol in the interfacial region. DMPC has an even lower ability to form an ordered phase than DPPC, and this ability is further reduced in the presence of propofol, which shows up as a decrease in the intensity of the water spectrum under all conditions in comparison with DPPC and DSPC, particularly at high surface pressures.

\subsection{NR experiments}

In order to learn about the location of propofol in DPPC monolayers spread on $0.89 \mathrm{mM}$ propofol solution and compressed to $5 \mathrm{mN} \mathrm{m}^{-1}$, we performed measurements using NR. First, pure lipid monolayers were measured and successfully modelled in four different isotopic contrasts using two stratified layers: alkyl tails with a volume fraction of 1 next to the air and hydrated head groups next to the water. These data and model fits, as well as a table of parameters used in the model, can be found in the Supporting Information. The area per lipid molecule, which can be calculated directly from the applied model, is consistent with that indicated by the corresponding surface pressure isotherm in Figure 2B $\left(\sim 80 \AA^{2}\right)$. 
Three different models were then applied to data from the mixed DPPC/propofol system, where propofol was located: (A) in the alkyl tails layer, (B) in the hydrated headgroups layer, and (C) under the hydrated headgroups in a separate third layer. The applications of these different models are shown in Figure 7. The best model fit to the experimental data, with good agreement in all four measured isotopic contrasts, occurs with propofol located in the hydrated headgroups layer (panel B). Transfer of propofol to the alkyl tails layer (panel A) results in an increase in reflectivity at mid-to-high $q_{z}$ of the model involving $\mathrm{d}_{62}$-DPPC in $\mathrm{D}_{2} \mathrm{O}$, which is not borne out in the experimental data. This feature evidences the need for an inversion in the scattering length density profile resulting from the propofol (low scattering length density) sitting between a dense layer of deuterated chains and the subphase (both high scattering length density), as highlighted by the arrow in the inset to panel B
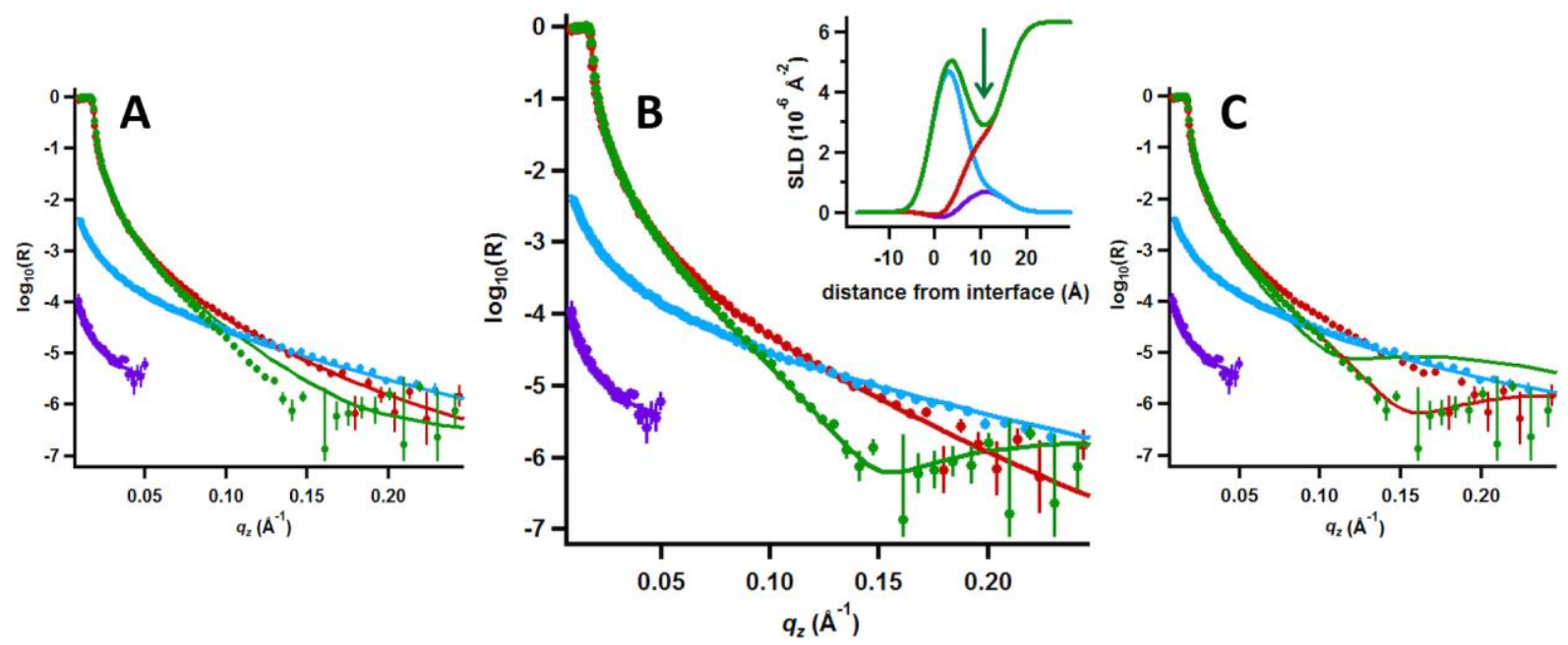

Figure 7. Experimental neutron reflectivity data and model fits of DPPC monolayers at $5 \mathrm{mN} \mathrm{m}^{-1}$ on $0.89 \mathrm{mM}$ propofol solution: (blue) $\mathrm{d}_{62}$-DPPC/ACMW, (green) $\mathrm{d}_{62}-\mathrm{DPPC} / \mathrm{D}_{2} \mathrm{O}$, (purple) DPPC/ACMW and (red) DPPC/ $\mathrm{D}_{2} \mathrm{O}$, where propofol is located A) in the alkyl tails layer, B) in the hydrated headgroups layer and C) under the hydrated headgroups layer. The inset to model B 
is the scattering length density (SLD) profiles of the data recorded in the 4 isotopic contrasts, and the green arrow marks the inversion in the profile discussed in the text.

On the other hand, transfer of propofol for inclusion beneath the hydrated headgroups layer (panel C) results in a Kiessig fringe at lower $q_{z}$ in the reflectivity model than occurs in the data in both contrasts involving $\mathrm{D}_{2} \mathrm{O}$. These features show that the material present at the interface is less extended than the structure of the 3-layer model.

It is interesting to note that the area per lipid molecule, again calculated directly from the applied model, is higher as a result of the propofol interaction than that indicated from the surface pressure isotherms in Figure 2B (156 vs. $\left.114 \AA^{2}\right)$. This difference indicates an increased solubilization of DPPC as a result of its interaction with the drug, and it may be noted that such a loss of phospholipid from a monolayer at the air/water interface has been observed also in the case of interactions with antimicrobial peptides ${ }^{39}$. See the Supporting Information. We note also that the location of propofol resolved using NR is consistent with the findings of Hansen ${ }^{17}$ et al. who demonstrated on pre-mixed propofol-DPPC monolayers that the drug was associated with the headgroups. However, as no $\mathrm{PO}_{2}$ frequency shift was detected using VSFS in the present work, we may infer that the interaction between propofol and the phosphate group of the lipid is weak.

\section{Conclusions}

Interactions between phospholipid monolayers and bilayers with surfactants have been extensively investigated during recent years and their destructive effect has been clearly recorded $^{1-4,67}$. Considerably less attention has been given to interactions between phospholipid layers and small amphiphilic non-surfactants. This is surprising since many important drug molecules fall into this molecular category ${ }^{4}$. In this article we address this general issue by 
considering the specific example of saturated phosphatidylcholines interacting with the drug propofol. To this end we utilize vibrational sum frequency spectroscopy (VSFS), neutron reflectometry and surface pressure isotherms to gain insight on where propofol is located and how it affects the molecular structure of the phospholipid monolayers DSPC, DPPC, and DMPC at the air-water interface. VSFS has previously been utilized for gaining insight on molecular order and hydration of phospholipids ${ }^{38,61,62}$ and surfactants ${ }^{67}$ at the air-water interface. This work thus extends these studies to include effects of amphiphilic non-surfactants on order and hydration of phospholipid monolayers.

Our data show that propofol is located in the phospholipid headgroup region at low surface pressures, but it is squeezed out of the monolayer as the phospholipid molecules pack closer together with increasing surface pressure. This effect was inferred from the surface pressure isotherms and then confirmed directly using VSFS and neutron reflectivity. It was demonstrated that propofol has the ability to increase the fluidity of the phospholipid monolayers, and this was enhanced for phospholipid monolayers possessing more disordered tails. Accordingly, DMPC was affected most, followed by DPPC, and DSPC was least affected by propofol. VSF spectra of the tail region show that disordered phospholipid monolayers become even more disordered in presence of propofol. The effect of propofol on the head group region depends on the area per molecule, which is affected by the phospholipid tail length. Propofol does not affect the headgroup of DSPC and DPPC at biological surface pressures, whereas at lower surface pressures as well as for the more disordered DMPC a reduction in signal is observed, which mainly depends on a lower lipid surface number density. The water surrounding the headgroups of a disordered monolayer is affected by the presence of propofol, while the water that is hydrating well-ordered monolayers is not. 
Taken together, our data suggest that propofol is incorporated preferentially in disordered phospholipid regions, which is consistent with computer simulations that provide evidence for accumulation of small amphiphilic compounds, like propofol, in such regions or at the border between the ordered and disordered regions ${ }^{45,46}$. Thus, provided the lipid layer is inhomogeneous with ordered and disordered domains, then one would also expect an inhomogeneous lateral distribution of propofol in the plane of a lipid membrane. Further studies along the lines presented here are needed to clarify the generality of our conclusions. Our findings also suggest that particular attention should be paid to studies devoted to determining the lateral distribution of amphiphilic molecules at the phospholipid-water interface in order to elucidate how lipid domain formations affect interactions with drugs and how drugs affect the phospholipid domains.

\section{SUPPORTING INFORMATION.}

Modeling approach and the parameters used in the NR work.

\section{ACKNOWLEDGEMENT}

This work was supported by Omya International AG. We thank the Institut Laue-Langevin for an allocation of beam time on FIGARO, Simon Wood for expert technical assistance, and the Partnership for Soft Condensed Matter for access to complementary techniques.

\section{References:}

1. Mitchell, D. J.; Ninham, B. W., Micelles, Vesicles and Microemulsions. Journal of the Chemical Society, Faraday Transactions 2: Molecular and Chemical Physics 1981, 77, 601-629. 2. Nazari, M.; Kurdi, M.; Heerklotz, H., Classifying Surfactants with Respect to Their Effect on Lipid Membrane Order. Biophysical Journal 2012, 102, 498-506.

3. Heerklotz, H., Interactions of Surfactants with Lipid Membranes. Quarterly Reviews of Biophysics 2008, 41, 205-264. 
4. Schreier, S.; Malheiros, S. V. P.; de Paula, E., Surface Active Drugs: Self-Association and Interaction with Membranes and Surfactants. Physicochemical and Biological Aspects. Biochimica et Biophysica Acta (BBA) - Biomembranes 2000, 1508, 210-234.

5. Vasileiou, I.; Xanthos, T.; Koudouna, E.; Perrea, D.; Klonaris, C.; Katsargyris, A.; Papadimitriou, L., Propofol: A Review of Its Non-Anaesthetic Effects. European Journal of Pharmacology 2009, 605, 1-8.

6. Zahn, M. D. Peter K.; Brennan, M. D. P. Timothy J., Physiologic and Pharmacologic Bases in Anesthesiology. The Journal of the American Society of Anesthesiologists 1997, 86, 751-751.

7. Trapani, G.; Altomare, C.; Sanna, E.; Biggio, G.; Liso, G., Propofol in Anesthesia. Mechanism of Action, Structure-Activity Relationships, and Drug Delivery. Current Medicinal Chemistry 2000, 7, 249-271.

8. Lugli, A. K.; Yost, C. S.; Kindler, C. H., Anaesthetic Mechanisms: Update on the Challenge of Unravelling the Mystery of Anaesthesia. European journal of anaesthesiology 2009, 26, 807-820.

9. $\quad$ Darvas, M.; Hoang, P. N. M.; Picaud, S.; Sega, M.; Jedlovszky, P., Anesthetic Molecules Embedded in a Lipid Membrane: A Computer Simulation Study. Physical Chemistry Chemical Physics 2012, 14, 12956-12969.

10. Osterman, J. E.; Hopper, J.; Heran, W. J.; Keane, T. M.; van der Kolk, B. A., Awareness


Psychiatry 2001, 23, 198-204.

11. Bogetz, M. D. Martin S.; Katz, M. D. Jeffrey A., Recall of Surgery for Major Trauma Anesthesiology 1984, 61, 6-9.

12. Giuseppe, M. T.; Cosimo, A.; Enrico, S.; Giovanni, B.; Gaetano, L., Propofol in Anesthesia. Mechanism of Action, Structure-Activity Relationships, and Drug Delivery. Current Medicinal Chemistry 2000, 7, 249-271.

13. Kotani, Y.; Shimazawa, M.; Yoshimura, S.; Iwama, T.; Hara, H., The Experimental and Clinical Pharmacology of Propofol, an Anesthetic Agent with Neuroprotective Properties. CNS Neuroscience \& Therapeutics 2008, 14, 95-106.

14. Krasowski, M. D.; Jenkins, A.; Flood, P.; Kung, A. Y.; Hopfinger, A. J.; Harrison, N. L., General Anesthetic Potencies of a Series of Propofol Analogs Correlate with Potency for Potentiation of $\Gamma$-Aminobutyric Acid (GABA) Current at the $\mathrm{GABA}_{\mathrm{A}}$ Receptor but Not with Lipid Solubility. Journal of Pharmacology and Experimental Therapeutics 2001, 297, 338-351.

15. Krasowski, M. D.; Hong, X.; Hopfinger, A. J.; Harrison, N. L., 4D-QSAR Analysis of a Set of Propofol Analogues: Mapping Binding Sites for an Anesthetic Phenol on the $\mathrm{GABA}_{\mathrm{A}}$ Receptor. Journal of medicinal chemistry 2002, 45, 3210-3221.

16. Yip, G.M.S.; Chen Z.W.; Edge C.J.; A Propofol Binding Site on Mammalian GABA Receptors Identified by Photolabeling. Nat. Chem. Biol. 2013, 9, 715-720.

17. Hansen, A. H.; Sørensen, K. T.; Mathieu, R.; Serer, A.; Duelund, L.; Khandelia, H.; Hansen, P. L.; Simonsen, A. C., Propofol Modulates the Lipid Phase Transition and Localizes near the Headgroup of Membranes. Chemistry and Physics of Lipids 2013, 175-176, 84-91.

18. Tsuchiya, H., Structure-Specific Membrane-Fluidizing Effect of Propofol. Clinical and Experimental Pharmacology and Physiology 2001, 28, 292-299.

19. Ali. M, Fauzia F., Jamal M., Synthesis, Spectroscopic, and in Vitro Cytotoxic Studies of Fatty Acid Analogues of 2, 6-Diisopropylphenol. Journal of Cancer Therapy 2010, 1, 124-130. 
20. Qian, K.; Shi, T.; Tang, T.; Zhang, S.; Liu, X.; Cao, Y., Preparation and Characterization of Nano-Sized Calcium Carbonate as Controlled Release Pesticide Carrier for Validamycin against Rhizoctonia Solani. Microchim Acta 2011, 173, 51-57.

21. Wacklin, H. P., Composition and Asymmetry in Supported Membranes Formed by Vesicle Fusion. Langmuir 2011, 27, 7698-7707.

22. Demel, R. A.; Geurts van Kessel, W. S. M.; Zwaal, R. F. A.; Roelofsen, B.; van Deenen, L. L. M., Relation between Various Phospholipase Actions on Human Red Cell Membranes and the Interfacial Phospholipid Pressure in Monolayers. Biochimica et Biophysica Acta (BBA) Biomembranes 1975, 406, 97-107.

23. Tyrode, E.; Niga, P.; Johnson, M.; Rutland, M. W., Molecular Structure Upon Compression and Stability toward Oxidation of Langmuir Films of Unsaturated Fatty Acids: A Vibrational Sum Frequency Spectroscopy Study. Langmuir 2010, 26, 14024-14031.

24. Niga, P., Self Assembly at the Liquid Air Interface: PhD Thesis, Stockholm 2010.

25. Hanahan, D. J., Handbook of Lipid Research; Plenum Press, 1978.

26. Mabrey, S.; Sturtevant, J. M., Investigation of Phase Transitions of Lipids and Lipid Mixtures by Sensitivity Differential Scanning Calorimetry. Proceedings of the National Academy of Sciences of the United States of America 1976, 73, 3862-3866.

27. Johnson, C. M.; Tyrode, E.; Baldelli, S.; Rutland, M. W.; Leygraf, C., A Vibrational Sum Frequency Spectroscopy Study of the Liquid-Gas Interface of Acetic Acid-Water Mixtures: 1. Surface Speciation. Journal of Physical Chemistry B 2005, 109, 321-328.

28. Shen, Y. R., Surface Properties Probed by Second-Harmonic and Sum-Frequency Generation. Nature 1989, 337, 519-25.

29. Campbell, R. A.; Wacklin, H. P.; Sutton, I.; Cubitt, R.; Fragneto, G., Figaro: The New Horizontal Neutron Reflectometer at the Ill. The European Physical Journal Plus 2011, 126, 107.

30. Braun, L.; Uhlig, M.; von Klitzing, R.; Campbell, R. A., Polymers and Surfactants at Fluid Interfaces Studied with Specular Neutron Reflectometry. Advances in Colloid and Interface Science 2017, 247, 130-148.

31. Lu, J. R.; Thomas, R. K.; Penfold, J., Surfactant Layers at the Air/Water Interface: Structure and Composition. Advances in Colloid and Interface Science 2000, 84, 143-304.

32. Nelson, A., Co-Refinement of Multiple-Contrast Neutron/X-Ray Reflectivity Data Using Motofit. Journal of Applied Crystallography 2006, 39, 273-276.

33. Yanez Arteta, M.; Campbell, R. A.; Nylander, T., Adsorption of Mixtures of Poly(Amidoamine) Dendrimers and Sodium Dodecyl Sulfate at the Air-Water Interface. Langmuir 2014, 30, 5817-5828.

34. Gaines, G. L., Insoluble Monolayers at Liquid-Gas Interfaces; Wiley (Interscience): New York, 1966, p 383.

35. Mitchell, M. L.; Dluhy, R. A., In Situ FT-IR Investigation of Phospholipid Monolayer Phase Transitions at the Air Water Interface. J. Am. Chem. Soc. 1988, 110, 712-18.

36. Hunt, R. D.; Mitchell, M. L.; Dluhy, R. A., The Interfacial Structure of Phospholipid Monolayer Films: An Infrared Reflectance Study. J. Mol. Struct. 1989, 214, 93-109.

37. Leonard-Latour, M.; Morelis, R. M.; Coulet, P. R., Interaction of Dmpc Liposomes with a Dmpa Monolayer: Related Study of Langmuir-Blodgett Films. Supramol. Sci. 1997, 4, 357363. 
38. Liljeblad, J. F. D.; Bulone, V.; Rutland, M. W.; Johnson, C. M., Supported Phospholipid Monolayers. The Molecular Structure Investigated by Vibrational Sum Frequency Spectroscopy. J. Phys. Chem. C 2011, 115, 10617-10629.

39. Jabłońska, A.; Ponikiewski, Ł.; Ejsmont, K.; Herman, A.; Dołęga, A., Syntheses, Spectroscopic and Structural Properties of Phenoxysilyl Compounds: X-Ray Structures, FT-IR and DFT Calculations. Journal of Molecular Structure 2013, 1054-1055, 359-366.

40. Ciumac, D.; Campbell, R. A.; Clifton, L. A.; Xu, H.; Fragneto, G.; Lu, J. R., Influence of Acyl Chain Saturation on the Membrane-Binding Activity of a Short Antimicrobial Peptide. ACS Omega 2017, 2, 7482-7492.

41. Liljeblad, J. F. D.; Bulone, V.; Tyrode, E.; Rutland, M. W.; Johnson, C. M., Phospholipid Monolayers Probed by Vibrational Sum Frequency Spectroscopy: Instability of Unsaturated Phospholipids. Biophysical Journal 2010, 98, L50-L52.

42. Guyot-Sionnest, P.; Hunt, J. H.; Shen, Y. R., Sum-Frequency Vibrational Spectroscopy of a Langmuir Film: Study of Molecular Orientation of a Two-Dimensional System. Physical Review Letters 1987, 59, 1597-600.

43. Bell, G. R.; Bain, C. D.; Ward, R. N., Sum-Frequency Vibrational Spectroscopy of Soluble Surfactants at the Air/Water Interface. Journal of the Chemical Society, Faraday Transactions 1996, 92, 515-23.

44. Liljeblad, J. D.; Rutland, M.; Bulone, V.; Johnson, C. M., The Structure of Model Membranes Studied by Vibrational Sum Frequency Spectroscopy. In Trends in Colloid and Interface Science Xxiii, Springer Berlin Heidelberg: 2010; Vol. 137, pp 9-12.

45. Mouritsen, O. G.; Jørgensen, K., Dynamical Order and Disorder in Lipid Bilayers. Chemistry and Physics of Lipids 1994, 73, 3-25.

46. Barnoud, J.; Rossi, G.; Marrink, S. J.; Monticelli, L., Hydrophobic Compounds Reshape Membrane Domains. PLoS Comput Biol 2014, 10, e1003873.

47. Casal, H. L.; Mantsch, H. H.; Paltauf, F.; Hauser, H., Infrared and 31P-NMR Studies of the Effect of $\mathrm{Li}^{+}$and $\mathrm{Ca}^{2+}$ on Phosphatidylserines. Biochim Biophys Acta 1987, 919, 275-86.

48. Casal, H. L.; Mantsch, H. H.; Hauser, H., Infrared Studies of Fully Hydrated Saturated Phosphatidylserine Bilayers. Effect of Lithium and Calcium. Biochemistry 1987, 26, 4408-16.

49. Chen, X.; Hua, W.; Huang, Z.; Allen, H. C., Interfacial Water Structure Associated with Phospholipid Membranes Studied by Phase-Sensitive Vibrational Sum Frequency Generation Spectroscopy. Journal of the American Chemical Society 2010, 132, 11336-11342.

50. Ma, G.; Allen, H. C., DPPC Langmuir Monolayer at the Air-Water Interface: Probing the Tail and Head Groups by Vibrational Sum Frequency Generation Spectroscopy. Langmuir 2006, 22, 5341-5349.

51. Pohle, W.; Selle, C.; Fritzsche, H.; Bohl, M., Comparative FTIR Spectroscopic Study Upon the Hydration of Lecithins and Cephalins. Journal of Molecular Structure 1997, 408-409, 273-277.

52. $\quad$ Patra, M.; Salonen, E.; Terama, E.; Vattulainen, I.; Faller, R.; Lee, B. W.; Holopainen, J.; Karttunen, M., Under the Influence of Alcohol: The Effect of Ethanol and Methanol on Lipid Bilayers. Biophysical Journal 2006, 90, 1121-1135.

53. Du, Q.; Superfine, R.; Freysz, E.; Shen, Y. R., Vibrational Spectroscopy of Water at the Vapor/Water Interface. Physical Review Letters 1993, 70, 2313-16.

54. Richmond, G. L., Molecular Bonding and Interactions at Aqueous Surfaces as Probed by Vibrational Sum Frequency Spectroscopy. Chemical Reviews 2002, 102, 2693-2724. 
55. Shultz, M. J.; Baldelli, S.; Schnitzer, C.; Simonelli, D., Aqueous Solution/Air Interfaces Probed with Sum Frequency Generation Spectroscopy. Journal of Physical Chemistry B 2002, 106, 5313-5324.

56. Ghosh, N.; Singh, A. K.; Mondal, J. A., pH Dependence of Interfacial Water in the Presence of Amino Acid Side Chains Revealed by Heterodyne-Detected Sum-Frequency Generation Spectroscopy. The Journal of Physical Chemistry C 2016, 120, 23596-23603.

57. Mondal, J. A.; Namboodiri, V.; Mathi, P.; Singh, A. K., Alkyl Chain Length Dependent Structural and Orientational Transformations of Water at Alcohol-Water Interfaces and Its Relevance to Atmospheric Aerosols. The Journal of Physical Chemistry Letters 2017, 8, 16371644.

58. Wen, Y.-C.; Zha, S.; Tian, C.; Shen, Y. R., Surface pH and Ion Affinity at the AlcoholMonolayer/Water Interface Studied by Sum-Frequency Spectroscopy. The Journal of Physical Chemistry C 2016, 120, 15224-15229.

59. Adhikari, A., Accurate Determination of Complex X(2) Spectrum of the Air/Water Interface. The Journal of Chemical Physics 2015, 143, 124707.

60. Yamaguchi, S., Development of Single-Channel Heterodyne-Detected Sum Frequency Generation Spectroscopy and Its Application to the Water/Vapor Interface. The Journal of Chemical Physics 2015, 143, 034202.

61. Watry, M. R.; Tarbuck, T. L.; Richmond, G. L., Vibrational Sum-Frequency Studies of a Series of Phospholipid Monolayers and the Associated Water Structure at the Vapor/Water Interface. The Journal of Physical Chemistry B 2003, 107, 512-518.

62. Walker, R. A.; Gragson, D. E.; Richmond, G. L., Induced Changes in Solvent Structure by Phospholipid Monolayer Formation at a Liquid-Liquid Interface. Colloids and Surfaces A: Physicochemical and Engineering Aspects 1999, 154, 175-185.

63. Allen, H. C.; Casillas-Ituarte, N. N.; Sierra-Hernandez, M. R.; Chen, X.; Tang, C. Y., Shedding Light on Water Structure at Air-Aqueous Interfaces: Ions, Lipids, and Hydration. Physical Chemistry Chemical Physics 2009, 11, 5538-5549.

64. Johnson, C. M.; Baldelli, S., Vibrational Sum Frequency Spectroscopy Studies of the Influence of Solutes and Phospholipids at Vapor/Water Interfaces Relevant to Biological and Environmental Systems. Chemical Reviews 2014, 114, 8416-8446.

65. Mondal, J. A.; Nihonyanagi, S.; Yamaguchi, S.; Tahara, T., Three Distinct Water Structures at a Zwitterionic Lipid/Water Interface Revealed by Heterodyne-Detected Vibrational Sum Frequency Generation. Journal of the American Chemical Society 2012, 134, 7842-7850.

66. Gragson, D. E.; Richmond, G. L., Probing the Intermolecular Hydrogen Bonding of Water Molecules at the Cc14/Water Interface in the Presence of Charged Soluble Surfactant. Journal of Chemical Physics 1997, 107, 9687-9690.

67. Tyrode, E.; Johnson, C. M.; Kumpulainen, A.; Rutland, M. W.; Claesson, P. M., Hydration State of Nonionic Surfactant Monolayers at the Liquid/Vapor Interface: Structure Determination by Vibrational Sum Frequency Spectroscopy. Journal of the American Chemical Society 2005, 127, 16848-16859.

68. Tyrode, E.; Johnson, C. M.; Rutland, M. W.; Claesson, P. M., Structure and Hydration of Poly(Ethylene Oxide) Surfactants at the Air/Liquid Interface. A Vibrational Sum Frequency Spectroscopy Study. Journal of Physical Chemistry C 2007, 111, 11642-11652. 
Table of Contents Image - TOC

Lipid condensed phase Lipid fluid phase

$3\} 3\} 3\}\}$

दर करत

Propofol

Propofol 